\title{
Neonatal oral administration of DiaPep277, combined with hydrolysed casein diet, protects against Type 1 diabetes in BB-DP rats. An experimental study
}

\author{
S. Brugman ${ }^{1}$ F. A. Klatter ${ }^{1}$ J. Visser ${ }^{2}$ N. A. Bos $^{1} \cdot$ D. Elias ${ }^{3} \cdot$ J. Rozing ${ }^{1}$ \\ ${ }^{1}$ Department of Cell Biology, Immunology Section, Groningen University, Groningen, The Netherlands \\ ${ }^{2}$ Department of Medical Microbiology, Molecular Virology Section, Groningen University, The Netherlands \\ ${ }^{3}$ Peptor, Rehovot, Israel
}

\begin{abstract}
Aims/hypothesis. Environmental factors such as diet and bacterial antigens play an important role in the onset of Type 1 diabetes. Different self-antigens are suggested to play a role in the development of diabetes. Antibodies against the $60-\mathrm{kDa}$ heat shock protein 60, which have a high homology to bacterial heat shock protein 65 , have been found in the circulation at the onset of diabetes in humans and in pre-diabetic NOD-mice. One of the immunodominant epitopes in autoimmune diabetes is $\mathrm{p} 277$, a specific peptide of human heat shock protein 60 corresponding to positions 437-460. In this study we investigated whether neonatal oral administration of DiaPep277 (a synthetic peptide analogue of p277) affected the development of diabetes in the BioBreeding-Diabetes Prone (BB-DP) rat, and whether this could potentiate the effect of a protective hydrolysed casein-diet.
\end{abstract}

Methods. BB-DP rats were orally inoculated once per day with placebo or DiaPep277 at days 4, 5, 6 and 7 of life. At the age of 21 days rats were weaned on to a conventional, cereal-based diet or on to the hydrolysed casein-diet.

Results. The development of diabetes in animals receiving DiaPep277 in combination with the hydrolysed casein-diet was delayed by 17 days, and a relative reduction of the incidence by $64 \%$ was seen. Nondiabetic animals did not show any sign of insulitis.

Conclusions/interpretation. Short-term neonatal feeding with p277 in early life, combined with diet adaptation, appears to provide a procedure to significantly reduce the development of Type 1 diabetes in later life.

Keywords BB-DP rat · DiaPep277 · Hydrolysed casein diet · Neonatal · Oral administration · Type 1 diabetes
Received: 5 January 2004 / Accepted: 19 April 2004 Published online: 10 July 2004

(C) Springer-Verlag 2004

\section{S. Brugman $(\varangle)$}

Department of Cell Biology, Immunology Section, Groningen University, A. Deusinglaan 1, 11th Floor, Room 1121, 9713 AV Groningen, The Netherlands E-mail: s.brugman@med.rug.nl

Tel.: +31-50-3632536, Fax: +31-50-3632512

Abbreviations: BB-DP, BioBreeding diabetes-prone · HC, hydrolysed casein $\cdot$ hsp60/65, heat shock protein $60 / 65$. ICA-69, islet cell antigen $69 \cdot \mathrm{NOD}$, non-obese diabetic

\section{Introduction}

Type 1 diabetes is an autoimmune disease that results from the destruction of the beta cells in the pancreas. Various self-antigens have been suggested to play a role in the development of diabetes. Indeed, antibodies against GAD, insulin, islet cell antigen (ICA-69), and heat shock protein 60 (hsp60) have been found in the circulation at the onset of diabetes in humans, and in animal models of diabetes $[1,2]$.

Oral tolerance induction to prevent autoimmune disease has been the topic of several studies. Oral GAD [3] and insulin, especially a B chain peptide, can prevent the development of Type 1 diabetes in nonobese diabetic (NOD) mice [4]. 
T cell reactivity against $\mathrm{p} 277$, a specific peptide of human hsp60, has been reported at the onset of diabetes in NOD mice [5]. Subcutaneous administration of p277 down-regulated T cell reactivity to beta cell antigens and prevented the development of diabetes in NOD mice. Treatment resulted in p277-specific IgG1 antibodies as well as in an increase of p277-specific IL-4 and IL-10 secretion and a decrease in gamma interferon secretion, suggesting an up-regulation of the T helper type 2 cytokine pathway [5].

In this study we investigated whether oral administration of DiaPep277 (a synthetic peptide analog of p277) affected the development of diabetes in neonatal BioBreeding diabetes-prone (BB-DP) rats, and whether this could potentiate the effect of a protective hydrolysed casein (HC) diet $[6,7,8]$.

\section{Materials and methods}

Experimental set-up. This experiment was designed to see if oral administration of a dominant epitope of hsp60 to neonatal animals could affect the development of Type 1 diabetes in the BB-DP rat model. Group-housed BB-DP rats (breeding colony, Groningen, the Netherlands) were orally inoculated once per day, either with placebo (distilled water) or with DiaPep277. Inoculation was done by silicone tube directly into the stomach, at days 4, 5, 6 and 7 of life. After weaning (age 21 days), rats received either a conventional, cereal-based diet ( $n=14$ placebo group, $n=16$ DiaPep277 group) (rodent diet no. Rmh-B2181, Hope Farms, Woerden, Netherlands) or an HC-diet ( $n=15$ placebo group, $n=16$ DiaPep277 group). The HC-diet is a modification of the AIN-93G diet containing $20 \%$ hydrolysed casein (Pancase S; Redstar Bioproducts, Tara, Ont, Canada) as the source of aminoacids, 53\% corn starch, $12 \%$ sucrose, 5\% corn oil, 5\% cellulose-type fibre (Solka-Floc; Teklad, Madison, Wis., USA) [3, 4]. Both sexes were used for this study. In our breeding colony 80 to $90 \%$ of the BB-DP rats spontaneously develop diabetes before the age of 130 days, with no differences between the sexes. Animals were treated in compliance with the Dutch law on experimental animal care. DiaPep277 [1] was kindly supplied by Peptor (Rehovot, Israel). Per inoculation, each rat was given $300 \mu \mathrm{g}$ of DiaPep277 in a volume of $300 \mu \mathrm{l}$. Body weights were measured three times per week. Animals that lost weight were screened for hyperglycaemia, using blood glucose strips (Roche Diagnostics, Almere, Netherlands). Rats were diagnosed as diabetic at plasma glucose levels above $15 \mathrm{mmol} / \mathrm{l}$. Non-diabetic animals were killed at the end of the study, at the age of 140 days.

Histopathology. Upon necropsy, the pancreas was removed, fixed in Bouin's solution, and after washing, embedded in paraffin. Sections of $7 \mu \mathrm{m}$ were stained with haematoxylin/eosin to evaluate insulitis. The degree of islet infiltration was measured using a Zeiss microscope and was rated as previously described [6] with the addition of score 5, which was developed in our lab to control for completely destroyed islets in severe insulitis. Per pancreas section, an average histological insulitis score was calculated by adding up the score of each islet and dividing it by the total number of islets counted. The analysis was performed blindly and independently by two persons.

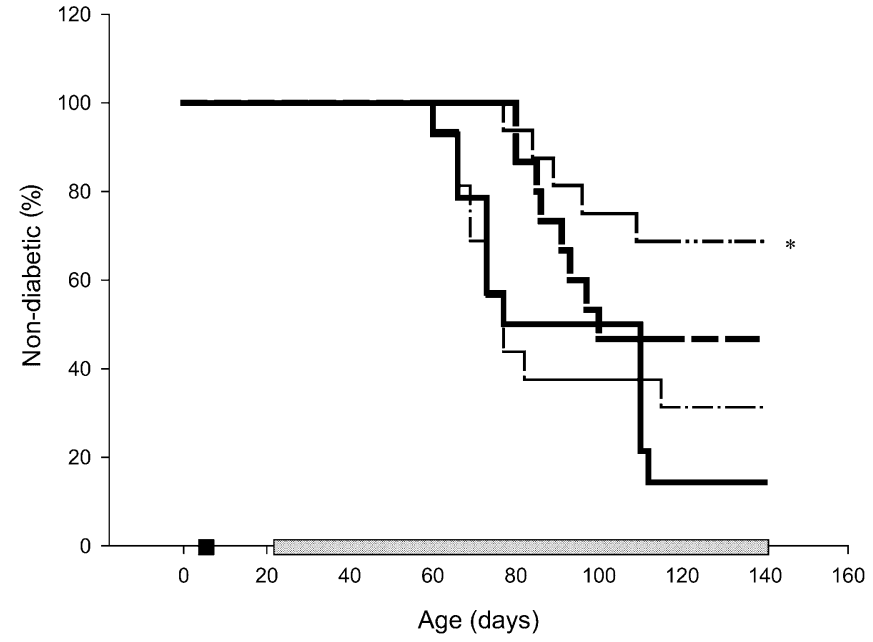

Fig. 1. Incidence of diabetes in the different treatment groups. The grey bar indicates time of exposure to diet, the black bar indicates duration of neonatal feeding with DiaPep277. Bold line: placebo + conventional diet $(n=14)$; broken bold line: placebo + HC-diet $(n=15)$; broken line with single dots: DiaPep277 + conventional diet $(n=16)$; broken line with double dots: DiaPep277 + HC-diet $(n=16)$. ${ }^{*} p=0.0034$ for DiaPep277 + HC-diet vs placebo + conventional diet, Kaplan-Meier, log rank test for survival

\section{Results}

As shown in Figure 1, administration of DiaPep277, combined with conventional rodent diet or an HC-diet from weaning, lowered the incidence of diabetes in BB-DP rats from $85 \%$ (placebo + conventional) to 69\% (DiaPep277 + conventional) and from 53\% (placebo $+\mathrm{HC}$ ) to $31 \%$ (DiaPep277 $+\mathrm{HC}$ ) respectively, although these differences were not significant. The HC-diet delayed the onset of diabetes by 20 days and tends to lower the incidence of diabetes from $85 \%$ to $53 \%$ (Kaplan-Meier, $p=0.06$ ).

In animals receiving DiaPep277 in combination with an HC-diet the onset of diabetes was delayed by 17 days and the incidence of diabetes was lower. In this group only $31 \%$ of the animals became diabetic (Kaplan-Meier; $p=0.0034$ vs placebo + conventional diet). All animals receiving DiaPep277 had a 2.2-fold improved chance of not developing diabetes (endpoint analysis, $\chi^{2}$ ).

The insulitis score of the different groups is depicted in Figure 2. Interestingly, the combination of neonatal administration of DiaPep277 followed by the HC-diet from weaning resulted in a dramatic reduction of insulitis in the non-diabetic rats (score approx. 1.0 , Wilcoxon signed rank; $p=0.043$ vs placebo + conventional diet). This score is comparable with scores found in healthy BB-DP rats and in diabetes-resistant $\mathrm{BB}$ rats with normal islet appearance. 


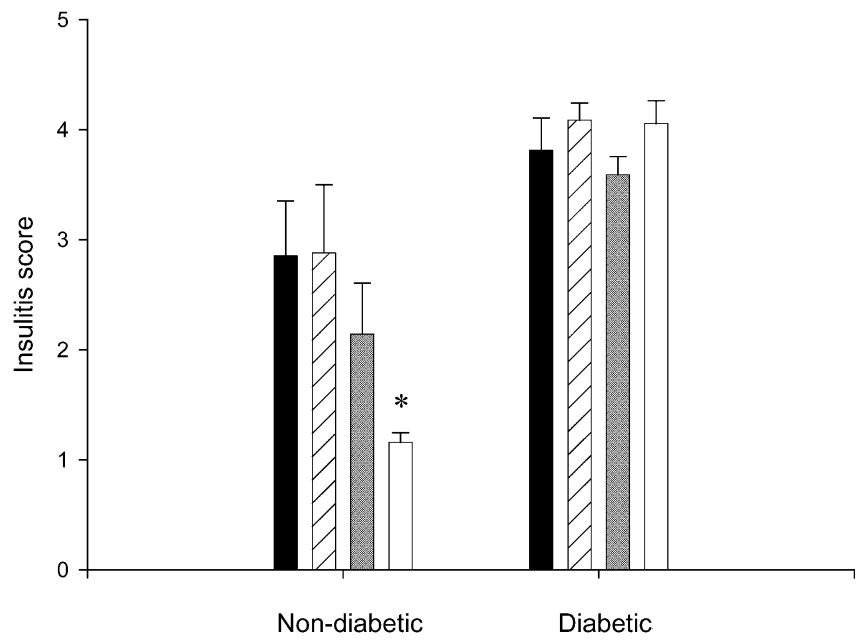

Fig. 2. Insulitis scores for non-diabetic and diabetic rats. Black bars: placebo + conventional diet $(n=14)$; hatched bars: DiaPep277 + conventional $(n=16)$; grey bars: placebo + HCdiet $(n=15)$; white bars: DiaPep277 + HC-diet $(n=16)$. Scores: 1, normal islet appearance; 2 , mild insulitis; 3 , severe insulitis; 4, end-stage islets; 5 , missing islets as compared to the average number of islets in the normal rat pancreas. * $p=0.043 \mathrm{com}$ pared with placebo + conventional diet, Wilcoxon signed rank

\section{Discussion}

In this study we demonstrate that a combination of DiaPep277 and HC-diet reduces the incidence of diabetes in BB-DP rats. This protection correlates with a lack of insulitis in the non-diabetic animals of this group.

Several other research groups have reported that an HC-diet has a protective effect on the development of diabetes in the BB-DP $[6,7,8]$. The protective effect of an HC-diet against diabetes could be the result of an actively induced protective mechanism or of nonexposure to diabetogenic substances present in plantbased rodent diets $[6,7]$.

The sequence of bacterial hsp65 has a high homology with human and rat hsp60. An immune response to hsp65 could lead to an autoimmune response to hsp60 on beta cells, due to molecular mimicry. Oral introduction of one of the epitopes of hsp60 to neonatal animals could lead to tolerance to this epitope, reducing the risk of autoimmunity induced by molecular mimicry, and thereby establishing protection at the level of the pancreatic beta cells. The administration of DiaPep277 alone in neonatal animals did not lead to significant protection against diabetes. Probably the overload of other diabetogenic epitopes, derived from plant-based diets or other bacterial antigens, masks the tolerance established for hsp60 and related proteins by oral administration of DiaPep277. Using a non-diabetogenic HC-diet could eliminate the masking effect.

In conclusion, we have shown that short-term neonatal feeding with DiaPep277 in early life, combined with diet modification, appears to provide a clinically relevant procedure to significantly reduce the risk of developing Type 1 diabetes later in life.

Acknowledgements. This work was supported by a grant from the Dutch Diabetes Foundation (project 2001.00.024).

\section{Conflict of interest}

D. Elias is an employee of Peptor Ltd. The company holds the license for developing DiaPep277 as a novel treatment in the prevention of Type 1 diabetes.

\section{References}

1. Cohen IR (2002) Peptide therapy for Type I diabetes: the immunological homunculus and the rationale for vaccination. Diabetologia 45:1468-1474

2. Yoon JW, Jun HS (2001) Cellular and molecular pathogenic mechanisms of insulin-dependent diabetes mellitus. Ann NY Acad Sci 928:200-211

3. Petersen JS, Karlsen AE, Markholst H, Worsaae A, Dyrberg T, Michelsen B (1994) Neonatal tolerization with glutamic acid decarboxylase but not with bovine serum albumin delays the onset of diabetes in NOD mice. Diabetes 44:1478-1484

4. Maron R, Guerau-de-Arellano M, Zhang X, Weiner HL (2001) Oral administration of insulin in neonates suppresses spontaneous and cyclophosphamide induced diabetes in the NOD mouse. J Autoimmun 16:21-28

5. Elias D, Meilin A, Ablamunits V et al. (1997) Hsp60 peptide therapy of NOD mouse diabetes induces a Th2 cytokine burst and down-regulates autoimmunity to various beta-cell antigens. Diabetes 46:758-764

6. Visser J, Brugman S, Klatter F et al. (2003) Short-term dietary adjustment with a hydrolysed casein-based diet postpones diabetes development in the diabetes prone $\mathrm{BB}$ rat. Metabolism 52:333-337

7. Scott FW, Cloutier HE, Kleemann R et al. (1997) Potential mechanisms by which certain foods promote or inhibit the development of spontaneous diabetes in BB rats. Diabetes 46:589-598

8. Mordes JP, Bortell R, Groen H, Guberski DL, Rossini AA, Greiner D (2001) Autoimmune diabetes mellitus in the BB rat. In: Sima AAF, Shafrir E (eds) Animal models for autoimmune diseases. Harwood, Amsterdam, pp 1-41 